\title{
Letter to Editor \\ Limitation of Double Disc Diffusion Method as a phenotypic confirmatory method for ESBL producing organisms in Clinical Microbiology Laboratory
}

\section{Thass N* and Randhawa VS}

Senior resident, Department of Microbiology, Lady Hardinge Medical College Director professor, Department of Microbiology, Lady Hardinge Medical College

\section{Dear Editor,}

In the recent years, Extended Spectrum $\beta$-lactamase (ESBL) producing gram negative bacteria are increasingly being reported worldwide .1 Extended spectrum $\beta$-lactamases are capable of conferring bacterial resistance to the penicillins, first, second, and third generation cephalosporins, and aztreonam (but not the cephamycins or carbapenems) by hydrolysis of these antibiotics. 2 Many Clinical Microbiology laboratories use double disc diffusion method to document ESBLs, which are subsequently reported to the clinicians. Performing genotypic tests for ESBLs in a routine microbiology laboratory is not feasible.

In the current study, we report atypical findings using double disc diffusion method that are of clinical relevance. CLSI 2018 has recommended phenotypic confirmatory tests for ESBLs, namely, the Double Disc Diffusion method which uses cefotaxime $(30 \mu \mathrm{g})$ or ceftazidime disks $(30 \mu \mathrm{g})$ with or without clavulanate $(10 \mu \mathrm{g})$ for phenotypic confirmation of of ESBLs. $3 \mathrm{~A}$ difference of $\geq 5 \mathrm{~mm}$ between the zone diameters of either of the cephalosporin disks and their respective cephalosporin/ clavulanate disk combination is taken to be phenotypic confirmation of ESBL production. We use this method in our laboratory for ESBL detection among members of enterobacteriaceae family.

*Author for Correspondence. E-mail: niveditathass1916@gmail.com www. ijamicro.com 
During the period of August 2018 to December 2018, blood culture samples of patients with suspected clinical sepsis were sent to our laboratory and eighty one isolates from these blood cultures were tested by this method at SSKH, a tertiary care hospital in North India. Of these, 22 got categorised as ESBLs. However, 32 isolates from patients revealed atypical results. The latter comprised of $10 \mathrm{E}$. coli and $22 \mathrm{~K}$. pneumoniae isolates. The ESBL screening revealed that these isolates showed no zone of inhibition with cefotaxime as well as cefotaxime plus clavulanate combination. These isolates appeared to be ESBLs as they showed no zone of inhibition with piperacillin-tazobactum, amoxicillin-clavulanate, cefepime and ceftazidime, but these did not get reported as ESBLs. The tests were repeated and same results were seen. Such isolates can be appropriately categorised by some genotypic method (a test available only in a few laboratories). Such atypical results may possibly be explained by co-existence of both enzyme types ( Amp C type $\beta$-lactamase and ESBL ) in the same strain .4 The AmpC-type $\beta$-lactamase resist inhibition by clavulanate and hence mask the synergistic effect of clavulanate and cephalosporins against ESBLs. 5

Missing out on such isolates may have a direct impact on therapeutic and infection control measures in the hospital. This can eventually compromise optimum patient antimicrobial therapy

\section{References:}

1. Nagdeo, NV., Kaore, NM., Thombare, VR., 2012, "Phenotypic methods for detection of various beta lactamases in gram negative clinical isolate: Need of the hour", Chron Young Sci, 3, pp. 2928 .

2. Taneja, N. and Sharma, M., 2008, "ESBLs detection in clinical microbiology: why \& how?," Indian J. Med. Res., 127, pp. 297-300.

3. Clinical and Laboratory Standard Institute. Performance standards for antimicrobial susceptibility testing: 29 ${ }^{\text {th }}$ ed CLSI supplement M100.Wayne, PA CLSI 2019.

4. Rawat, D. and Nair, D.,2010, "Extended-spectrum $\beta$-lactamases in Gram Negative Bacteria", J. Glob. Infect. Dis., 2 (suppl 3), pp. 263-274.

5. Munier et al., 2010, "Positive Extended-Spectrum- $\beta$-Lactamase (ESBL) Screening Results May Be Due to AmpC $\beta$-Lactamases More Often than to ESBLs", Journal of Clinical Microbiology, 48 (suppl 2), pp. 673-674. 